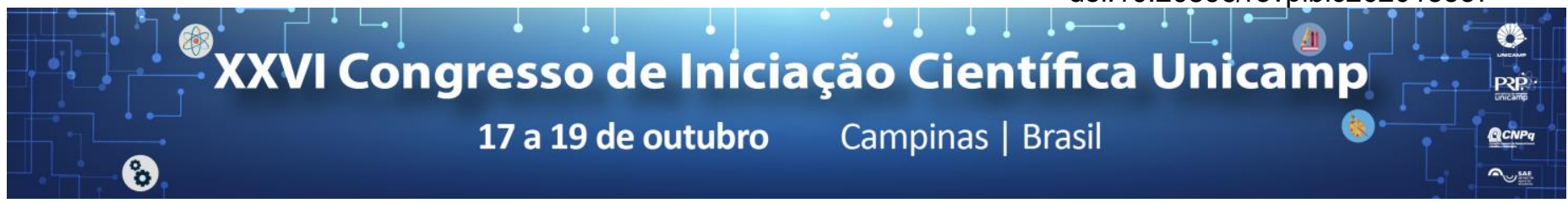

\title{
Nitric oxide releasing collagen membranes for the topical treatment of wounds
}

\author{
Giovanna J. V. P. dos Santos*; Guilherme F. Picheth; Valéria Póvoa; Eliana P. de Araujo; Marcelo G. de \\ Oliveira.
}

\section{Abstract}

Nitric oxide (NO) donor biomaterials have great potential for the promotion of tissue regeneration. Collagen membranes impregnated with S-nitrosoglutathione (GSNO), an NO donor, were developed, characterized and applied in vivo, demonstrating the acceleration of wound healing.

\section{Key words:}

biomaterials, collagen, nitric oxide

\section{Introduction}

The use of dressings derived from collagen is already established in the medical area for the coating of lesions and healing aid. Nitric oxide (NO) is directly involved in angiogenic and proliferative activities, stimulating the tissue repair process in healthy individuals or patients suffering from chronic diseases (e.g. diabetes). The main objective of this project is the development of type I collagen membranes functionalized with S-nitrosoglutathione (GSNO) for the localized release of NO.

\section{Results and Discussion}

Collagen membranes were characterized by FTIR, SEM/EDS. The NO release of the membranes was characterized by chemiluminescence.
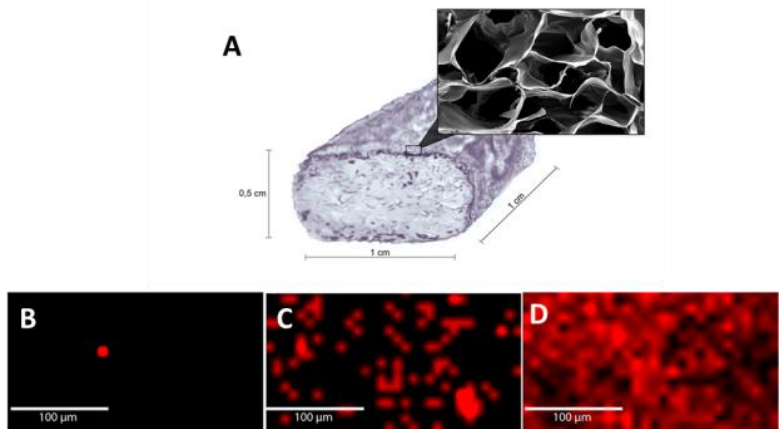

Image 1. (A) Macroscopic photograph of the membrane with details of the internal structure of macropores obtained by SEM. (B, C and D) Sulfur mapping of native collagen (B), collagen with dose 1 of GSNO (C) and dose 2 of GSNO (D) (dose 2>dose 1).

The NO donor collagen membranes displayed high porosity (IMG. 1) and NO release kinetics dependent on the hydration degree (IMG. 2).

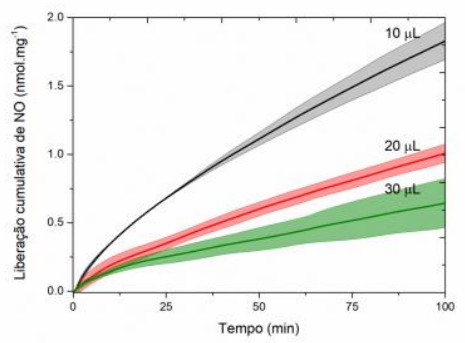

Image 2. Kinetic curves of NO released from collagen/GSNO membranes swollen with $10 \mu \mathrm{L}, 20 \mu \mathrm{L}$ and $30 \mu \mathrm{L}$ of PBS with EDTA.
In vivo experiments showed a higher wound healing rate after 4, 6 and 8 days (IMG. 3), as well as larger amounts of cytokines with proliferative activity (e.g. IGF-1 and SDF-1), and overexpression of iNOS and MMP-9 in animals treated with collagen/GSNO membranes.

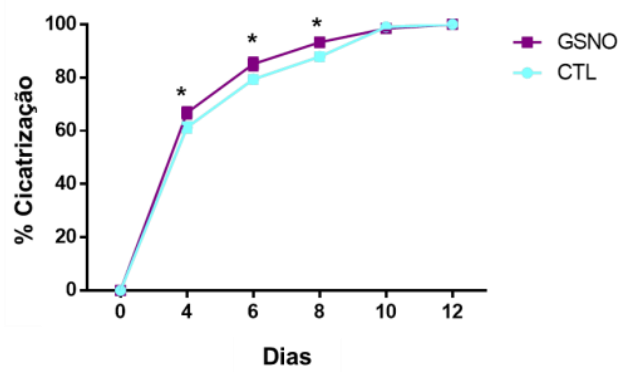

Image 3. Evolution of the lesion area of animals treated with collagen/GSNO membranes (GSNO) and native collagen membrane (CTL), over 12 days.

\section{Conclusions}

The collagen membranes impregnated with GSNO accelerated cell proliferation and wound healing possessing potential for the treatment of chronic wounds.

\section{Acknowledgement}

GJVPS received a studentship from the São Paulo Research Foundation (FAPESP) (Grant no 2017/046150). This work was supported by FAPESP (grant number 2016/02414-5).

${ }^{1}$ Champeau, M., Póvoa, V., Militão, L., Cabrini, F. M., Picheth, G. F., Meneau, F., Jara, C. P., de Araujo E. P., de Oliveira, M. G. (2018). Supramolecular poly (acrylic acid)/F127 hydrogel with hydration-controlled nitric oxide release for enhancing wound healing. Acta biomaterialia, 74, 312 325 .

${ }^{2}$ Shoulders, M. D., \& Raines, R. T. (2009). Collagen structure and stability. Annual review of biochemistry, 78, 929-958. 